\title{
Corrigendum: SVInterpreter: A Comprehensive Topologically Associated Domain Based Clinical Outcome Prediction Tool for Balanced and Unbalanced Structural Variants
}

\author{
Joana Fino $^{1 *}$, Bárbara Marques ${ }^{1}$, Zirui Dong ${ }^{2,3,4}$ and Dezso David ${ }^{1 *}$ \\ ${ }^{1}$ Department of Human Genetics, National Health Institute Doutor Ricardo Jorge, Lisbon, Portugal, ${ }^{2}$ Department of Obstetrics and \\ Gynaecology, The Chinese University of Hong Kong, Hong Kong, China, ${ }^{3}$ Shenzhen Research Institute, The Chinese University of \\ Hong Kong, Shenzhen, China, ${ }^{4}$ Hong Kong Hub of Pediatric Excellence, The Chinese University of Hong Kong, Hong Kong, China
}

Keywords: SVInterpreter, bioinformatic web-tool, clinical outcome prediction, balanced structural variants, copy number variants, topologically associated domains, phenotypic comparison

\section{OPEN ACCESS}

Approved by:

Frontiers Editorial Office,

Frontiers Media SA, Switzerland

*Correspondence:

Joana Fino

joana.fino@insa.min-saude.pt

Dezso David

dezso.david@insa.min-saude.pt

Specialty section:

This article was submitted to Human and Medical Genomics,

a section of the journal

Frontiers in Genetics

Received: 02 February 2022 Accepted: 07 February 2022 Published: 25 February 2022

Citation:

Fino J, Marques B, Dong Z and David D (2022) Corrigendum: SVInterpreter: A Comprehensive

Topologically Associated Domain Based Clinical Outcome Prediction Tool for Balanced and Unbalanced

Structural Variants.

Front. Genet. 13:868306. doi: 10.3389/fgene.2022.868306

\section{A Corrigendum on}

SVInterpreter: A Comprehensive Topologically Associated Domain-Based Clinical Outcome Prediction Tool for Balanced and Unbalanced Structural Variants

by Fino, J., Marques, B., Dong, Z., and David, D. (2021). Front. Genet. 12:757170. doi: 10.3389/fgene. 2021.757170

In the original article, there was an error. The comparison with AnnotSV was made with its first version, which is currently out of date. Any remark made on this tool should not be considered.

The versions, commits and release dates of the tools used for comparison were not indicated.

Therefore, a correction has been made to Discussion, paragraph 11:

"Comparatively with other recent tools that support the evaluation of SVs, such as position_effect (commit: fced2c49, 13 June 2017), AnnotSV (Version 1.0, 21 December 2017) and ClinTAD (commit: 09b4925fb, 18 September 2019), SVInterpreter seems to be more comprehensive (ZepedaMendoza et al., 2017; Geoffroy et al., 2018; Spector and Wiita, 2019). First, SVInterpreter showed to be the one that allows more customization and adjustments, since, for example, AnnotSV and ClinTAD only work with one genome version, and ClinTAD only uses TAD boundaries of human embryonic stem cell data. Then, SVInterpreter shows a broader view of the affected regions, accounting for both gene disruption and position effects: AnnotSV is focused on the identification of genes directly affected by a breakpoint, and position_effect was designed to identify candidate genes essentially from position effect events. In regard to phenotypic comparison, as AnnotSV does not perform any, and ClinTAD is limited to the full HPO term overlap, position_effect is the only one with a similar functionality. Also, SVInterpreter is the one that retrieves the most information, including the position effect important data, GeneHancer cluster of interactions and chromatin loops, phenotypic data from DDG2P and clinGen, Gene-phenotype/disease associations in animal models, and GWAS data. Therefore, the existence of overlooked information by position_effect and AnnotSV, as shown 
in DGAP107, may contribute to limited results, biased candidate gene prioritization, and the need of additional resources."

The authors apologize for the errors and state that this does not change the scientific conclusions of the article in any way. The original article has been updated.

Publisher's Note: All claims expressed in this article are solely those of the authors and do not necessarily represent those of their affiliated organizations, or those of the publisher, the editors and the reviewers. Any product that may be evaluated in this article, or claim that may be made by its manufacturer, is not guaranteed or endorsed by the publisher.

Copyright (c) 2022 Fino, Marques, Dong and David. This is an open-access article distributed under the terms of the Creative Commons Attribution License (CC BY). The use, distribution or reproduction in other forums is permitted, provided the original author(s) and the copyright owner(s) are credited and that the original publication in this journal is cited, in accordance with accepted academic practice. No use, distribution or reproduction is permitted which does not comply with these terms. 